\title{
ECS-induced retrograde amnesia is not due to increased sensitivity to sources of ordinary forgetting
}

\author{
RALPH R. MILLER, WESLEY J. KASPROW, and TODD R. SCHACHTMAN \\ State University of New York at Binghamton, Binghamton, New York
}

\begin{abstract}
A series of experiments was conducted with rats to determine if the amount of potentially interfering information presented to a rat immediately following training and electroconvulsive shock (ECS) influenced the magnitude of ECS-induced amnesia. Using one-trial passive avoidance, multitrial active avoidance, one-trial active approach, and appetitive delayed alternation tasks, ECS intensity thresholds were established for each task (Experiments 1, 3, 5, and 7, respectively). Then some subjects were exposed to task-irrelevant high environmental stimulation (of low affective value) immediately following ECS of an intensity that was normally just below the amnestic threshold. Simultaneously, other subjects were exposed to sensory isolation immediately following ECS of an intensity that was normally just above the amnestic threshold (Experiments $2,4,6$, and 7 , respectively). The posttraining environment in the absence of ECS had no effect on passive avoidance, a nonsignificant tendency towards an effect on active avoidance and active approach, and a strong effect on delayed alternation. When the posttraining environment did have an effect, high stimulation impaired retention and sensory isolation enhanced retention. In no case was there evidence that retroactive stimulus interference was more than algebraically additive with respect to ECS-induced amnesia.
\end{abstract}

Historically, electroconvulsive shock (ECS) and similarly acting amnestic agents such as hypothermia were thought to produce retrograde amnesia (RA) by interfering with ongoing electrochemical neural transmission that served to uniquely encode newly acquired information before the information was consolidated into the less labile structural/chemical format of long-term retention (e.g., McGaugh, 1966). However, recently observed phenomena have rendered this view untenable (for a review, see Miller \& Marlin, 1979). Given the now well established reversibility of at least part, if not all, RA (e.g., Miller \& Springer, 1972), RA is frequently referred to as a form of retrieval failure (Spear, 1978). However, even if the retrieval-failure hypothesis is accepted as valid, the underlying mechanisms for RA remain to be identified.

Although several physiological explanations of RA have been proposed as alternatives to the consolidation-failure position (e.g., Gold \& Sternberg, 1978), these alternative explanations have not been able to address all the phenomena that were originallly cited in support of the consolidation-failure position. As a first step in attempting to remedy these deficiencies, Deutsch (1973) proposed a psychological explanation of ECS-induced RA, posit-

This research was supported by NIMH Grant 33881 . Gratitude is expressed to David Daniel for his assistance in collecting the data, to Andrea Brown for her critiquing an early version of the manuscript, and to Roxanne Vavra for her typing of the manuscript.

The authors' mailing address is: Department of Psychology, SUNYBinghamton, Binghamton, NY 13901. ing that ECS temporarily increases an animal's susceptibility to "normal" environmental sources of forgetting. This view is potentially an improvement over the original consolidation-failure hypothesis in that it is compatible with many of the phenomena that create problems for the consolidation position. For example, reminder-induced recovery from RA is paralleled by reminder-induced recovery from "normal" forgetting (Smith \& Spear, 1979). Obviously, Deutsch's rather molar proposal is not a substitute for a physiologically based explanation of RA, but its confirmation would be a strong incentive for further cross-fertilization between information processing models and physiological analysis of both RA and "normal" forgetting.

Deutsch's (1973) suggestion that ECS potentiates other sources of forgetting has appeal owing to its apparent consistency with many ECS-related phenomena. However, there are few data to support this radical view, in contrast to the more traditional position that ECS is itself a primary source of forgetting. Among the limited supportive data, Peters, Calhoun, and Adams (1973) report that, following one-trial appetitive training and ECS, sensory isolation attenuated ECS-induced RA in rats. However, the interpretation of this observation is clouded by Calhoun, Prewett, Peters, and Adam's (1975) observation that, using one-trial passive avoidance and ECS with rats, darkness alone, rather than sensory isolation, during the recovery period appeared to be the critical factor in attenuating ECS-induced amnesia. Moreover, Adams, Calhoun, Davis, and Peters (1974), using rats in a multitrial 
active avoidance task, found that post-ECS sensory isolation (including darkness) enhanced RA. Despite these problems, some support for Deutsch's (1973) hypothesis might be seen in a report by Hinderliter, Smith, and Misanin (1976), who, using rats in a one-trial passive avoidance task, found that sensory isolation (including darkness) reduced RA even if the isolation occurred only immediately after ECS rather than throughout the retention interval as was done by Calhoun et al. One problem with such an interpretation of their study is that postacquisition sensory isolation without preceding ECS attenuated, rather than enhanced, performance relative to appropriate controls. Moreover, neither in any of the studies performed by Adams and his colleagues nor in the Hinderliter et al. study were the animals given pretraining familiarization to the sensory-isolation environment. Thus, although their isolation enclosures provided attenuated stimulation relative to the normal colony room, the enclosures themselves were new to the subjects at the time of treatment and consequently were a potential source of novel information that could compete with the target information for the presumably limited information processing capacity of the animal (Miller, Greco, Marlin, \& Balaz, in press). The following experiments were intended as a more decisive test of Deutsch's hypothesis than are these prior studies, which were not meant to be explicit tests of Deutsch's suggestion. On a less theoretical level, the present research asked if unusually high or low environmental stimulation immediately following ECS would alter the magnitude of observed RA.

\section{EXPERIMENT 1}

Following the lead of Calhoun et al. (1975) and Hinderliter et al. (1976), we used a passive avoidance task to examine the effects of post-ECS sensory stimulation on RA. Unlike these prior studies, we proposed to observe the effects of increased stimulation (relative to the animal colony) as well as decreased stimulation. To maximize sensitivity to possible effects of post-ECS sensory stimulation, we felt it essential that our sensory-isolation animals receive an ECS of the minimal intensity necessary to produce RA without sensory isolation; any stronger ECS might produce a ceiling effect that would mask the action of post-ECS sensory isolation. Similarly, we thought it necessary that our post-ECS high-stimulation animals receive an ECS of an intensity just below the threshold for producing RA in animals lacking the high sensory stimulation; any weaker ECS might result in a floor effect that would mask the action of post-ECS high stimulation. (We considered preceding sensory isolation and high sensory stimulation with a common value of ECS that produced intermediate RA, but rejected the possibility because modest levels of ECS resulted in half the animals' displaying considerable RA and half the animals' displaying little RA, rather than all the animals' displaying modest RA. That is, modest ECS yielded highly variable performance.) Thus, our first study was designed to determine appropriate intensities of ECS given our particular training parameters. No controls for the associative nature of the observed RA were included, since the purpose of Experiment 1 was not to prove that RA reflected an associative failure as opposed to a systemic, stimulus-nonspecific change in behavior, but to determine the ECS-intensity threshold for RA. [Other investigators (e.g., McGaugh, 1966) have reviewed abundant data demonstrating the associative nature of ECS-induced RA.].

\section{Method}

Subjects. Forty naive male Sprague-Dawley-derived rats weighing between 195 and $295 \mathrm{~g}$ served as subjects. These animals were individually housed in standard suspended stainless steel wire-mesh cages. To minimize the probability of ECS-induced back injuries, the subjects were kept lean by being restricted to $10 \mathrm{~g}$ per day of powdered Purina Laboratory Chow starting 1 week before the beginning of the experiment. On experimental days, food was given approximately $1 \mathrm{~h}$ after the experimental manipulation. Water was available ad lib. The lights in the animal colony were on $24 \mathrm{~h}$ per day. Each animal was handled, just prior to feeding, for $30 \mathrm{sec}$ daily during the week before initiation of the experiment.

Apparatus. Training and testing occurred in a step-down apparatus. The stainless steel grid floor of the apparatus measured 50.8 $\times 50.8 \mathrm{~cm}$ and was enclosed on all four sides by opaque $57.2 \mathrm{~cm}-$ high walls. A $17.1 \times 9.5 \mathrm{~cm}$ wire-mesh platform was centered $9 \mathrm{~cm}$ above the grid floor. A pair of earclip electrodes made from modified minigator clips were suspended from a counterweighted pulley system mounted directly over the platform. The experimental room was dimly illuminated by a shaded $25-\mathrm{W}$ light bulb, and a 63-dBC (re $20 \mu \mathrm{N} / \mathrm{m}^{2}$ ) white noise provided auditory masking. The grid floor was wired to deliver a $1-\mathrm{sec}, 1.3-\mathrm{mA}, 60-\mathrm{Hz}$ scrambled constant-current footshock effectively scrambled by a daisy chain of NE-2 neon bulbs. The earclips were wired to deliver a $300-\mathrm{msec}$, $60-\mathrm{Hz}$ constant-current ECS of variable intensity (see Procedure section).

Procedure. On the training day, each animal was carried into the experimental room, had earclips attached, and was placed on the platform facing away from the experimenter. Latency for each rat to place all four paws on the grid floor was recorded (range $=12$ to $107 \mathrm{sec}$ ). Three seconds later, the animal was returned to its home cage. Two subjects were replaced with equivalent animals due to excessive fighting to free themselves from the earclips. Ninety minutes later, this procedure was repeated (range of latencies $=6$ to $42 \mathrm{sec}$ ). A third trial was given $90 \mathrm{~min}$ after the second (range $=3$ to $37 \mathrm{sec}$ ). All subjects were then assigned to one of five groups $(n=8)$, counterbalancing for both Trial 3 stepdown latencies and body weights. Ninety minutes after Trial 3, a fourth trial was given. When all four paws touched the grid floor (range $=2$ to $39 \mathrm{sec}$ ), footshock was administered, followed immediately by ECS of 0-, 30-, 40-, 55-, or 80-mA intensity. After ECS, all subjects were returned to their home cages. Sixty-eight hours later, each subject was returned to the platform without earclips and step-down latencies were recorded. A 10-min ceiling was imposed on test latencies. Five animals reached this ceiling, three in Group 0, and two in Group 30.

\section{Results and Discussion}

All latencies were transformed into log latencies to improve the normality of the within-group distributions. Retention, as indexed by step-down latency for each group, is illustrated in Figure 1. Clearly, retention decreased as ECS intensity increased $[\mathrm{F}(4,34)=27.60$, $\mathrm{p}<.01]$. This positive correlation between ECS inten- 


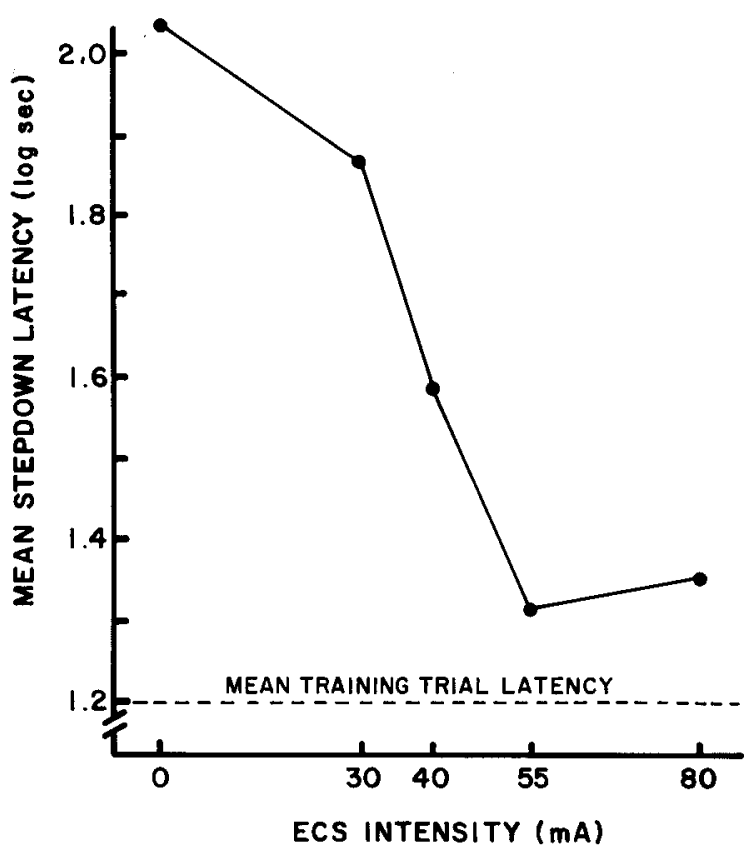

Figure 1. Mean step-down latency as a function of ECS intensity following one-trial passive avoidance training. The dotted line represents the mean training trial latency based on all animals.

sity and degree of RA has been found to hold across a wide variety of tasks (e.g., Pagano, Bush, Martin, \& Hunt, 1969) and consistently appears throughout the subsequent studies in this series. Inspection of Figure 1 suggests that, with the present passive avoidance task parameters, a 30-mA ECS is just below the threshold for producing robust RA and a 55-mA ECS is just above the threshold for inducing RA. These latter two groups did differ from one another $[\mathrm{t}(15)=2.48, \mathrm{p}<.05]$.

\section{EXPERIMENT 2}

Using the results of Experiment 1, we now asked if post-ECS stimulus isolation would attenuate RA in animals given ECS just above the threshold for RA (ECS-55). Simultaneously, we exposed animals to $1 \mathrm{~h}$ of high environmental stimulation immediately following the ECS in an effort to induce RA in animals given ECS just below the threshold for RA (ECS-30). For purposes of comparison, additional animals were exposed to one or the other of the ECS intensities but spent the entire post-ECS interval in their home cages. Moreover, to determine the direct effects of the posttraining high- and low-stimulation environments upon performance independent of interactions with ECS, animals were included that received posttraining low, medium (home cage), or high stimulation without ECS.

\section{Method}

Subjects and Apparatus. Forty-two rats, identical to those in Experiment 1 except that their starting weights were between 195 and $370 \mathrm{~g}$, served as subjects. In addition to the apparatus used in Experiment 1, three low- and three high-stimulation enclosures located in an adjacent room were employed. The low-stimulation enclosure consisted of the animal's home cage (without food dish or water bottle) in an unilluminated industrial Acoustics Corp. smallanimal, sound-attentuating (23-dBC white noise, re $20 \mu \mathrm{N} / \mathrm{m}^{2}$ ), chamber. This chamber was subdivided into three compartments with acoustic tiles, such that the enclosure could hold three animals simultaneously in isolation from one another. The bedding in the low-stimulation chamber was changed after each use by each animal in order to minimize olfactory stimulation. The high-stimulation chambers consisted of three suspended cylindrical wooden boxes, $45 \mathrm{~cm}$ in diameter and $15 \mathrm{~cm}$ high, with cedar chips on the floor and an unfamiliar male albino rat (230-290 g) present; moreover, tape-recorded popular music (the same for each animal) was played ( $82 \mathrm{dBC}$ maximum), the $75-\mathrm{W}$ room light that penetrated the many screened air holes in the cylindrical enclosure flashed $0.8 \mathrm{sec}$ on/ $0.2 \mathrm{sec}$ off, and the boxes were gently swung by a variable-speed motor in a horizontal figure-eight pattern $(30 \times 10 \mathrm{~cm})$ that was completed once every $4.1 \mathrm{sec}$. The high-stimulation context was chosen on the basis of pilot studies indicating that it elicited prolonged exploratory behavior without evidence of great fear, that is, freezing or defecation.

Procedure. With the following exceptions, the procedure was the same as in Experiment 1 . On the 3 days immediately preceding training, each animal was placed in the sensory-isolation enclosure for $15 \mathrm{~min}$. This manipulation was intended to reduce the sensory stimulation provided by the enclosure's novelty on the training day. After Trial 1, three animals were replaced due to excessive efforts to remove the earclips. After the third step-down trial, all subjects were assigned to one of seven groups $(n=6)$ counterbalanced for body weight and Trial 3 latencies. On Trial 4, all animals received footshock upon stepping off the platform. This footshock was followed with 0-mA ECS for three groups, 30-mA ECS for two groups, and 55-mA ECS for the remaining two groups. We followed the example of Hinderliter et al. (1976) in providing $1 \mathrm{~h}$ of modified stimulation after ECS. Consequently, after ECS, one 55-mA-ECS group was placed in the low-stimulation enclosure for $1 \mathrm{~h}(55-\mathrm{LS})$ and the other 55-mA-ECS group was immediately returned to the colony (55-C). One 30-mA-ECS group was placed in the high-stimulation enclosure for $1 \mathrm{~h}(30-\mathrm{HS})$ and the other 30 $\mathrm{mA}-\mathrm{ECS}$ group was immediately returned to the colony (30-C). Of the three groups receiving no ECS $(0-\mathrm{mA})$, one was placed in the low-stimulation enclosure for $1 \mathrm{~h}$ after training (0-LS), one was placed in the high-stimulation enclosure for $1 \mathrm{~h}$ after training (0HS), and the last group was immediately returned to the colony after training (0-C). As in Experiment 1, testing occurred approximately $68 \mathrm{~h}$ later. The experiment was run in two balanced replications.

\section{Results and Discussion}

No differences were found between replications, so the data from the two replications were pooled. Group mean test latencies are illustrated in Figure 2. Consistent with Experiment 1, in which animals were returned to their home cages immediately after ECS, 55-mA ECS produced significantly more RA in Group 55-C than 30-mA ECS produced in Group 30-C [t(11) = 3.08, p < .02] and 30mA ECS yielded a nonsignificant tendency towards more RA in Group 30-C than 0-mA ECS yielded in Group 0$\mathrm{C}(.2>\mathrm{p}>.1)$. However, no significant differences or even suggestive tendencies were found between groups receiving the same intensity of ECS (all ps $>.5$ ). Although there were only six subjects per group, the means of groups receiving the same intensity of ECS were 


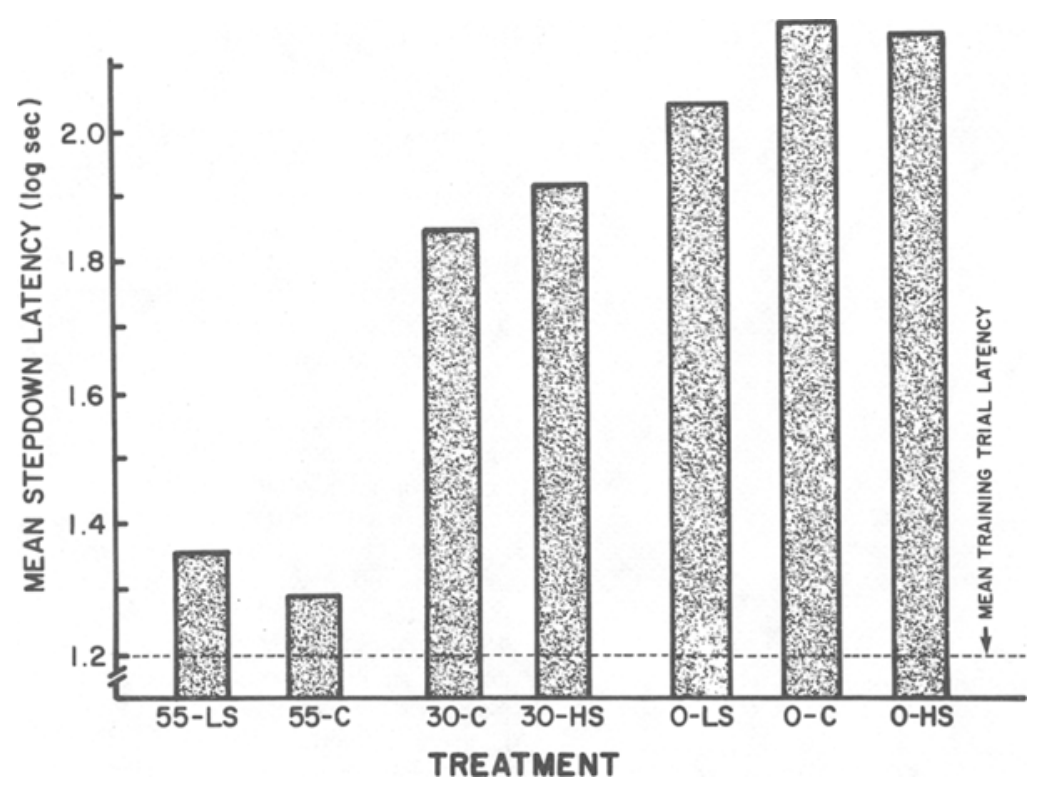

Figure 2. Mean step-down latency after one-trial passive avoidance training as a function of ECS intensity (0, 30, or $55 \mathrm{~mA}$ ) and post-ECS environment (LS = low stimulation, $\mathrm{C}=$ home cage in animal colony, $\mathrm{HS}=$ high stimulation).

so close that a power analysis indicated significance would not be reached even if the number of animals per group were increased tenfold. Moreover, the nonsignificant tendency towards high-stimulation's attenuating RA in 30$\mathrm{mA}$-ECS animals was in the direction opposite to the effect predicted by Deutsch's (1973) hypothesis, thereby balancing the nonsignificant tendency in support of Deutsch's hypothesis seen in the 55-mA-ECS, lowstimulation animals.

Overall, there is no suggestion in the present data that post-ECS stimulation modulates RA in animals receiving ECS. Thus, the current data may be viewed as unsupportive of Deutsch's (1973) hypothesis that ECS, rather than being a source of memory degradation in its own right, increases sensitivity to "normal" sources of timedependent forgetting. However, such a conclusion is rendered tenuous by the lack of effect of either the high- or low-stimulation condition upon animals that received no ECS, an observation consistent with previous passive avoidance data reported by Marlin, Greco, and Miller (1978). Possibly this lack of effect is due to a ceiling effect of some sort on test scores; however, this is not highly likely, since only 3 of the 18 animals in no-ECS groups failed to step down during the full 10 min allowed. Consequently, the possibility exists that ECS may increase sensitivity to other sources of forgetting but that those sources of forgetting in the present passive avoidance preparation were not sufficiently strong to influence behavior. Since a more effective way to increase the impact of our low-and high-stimulation treatments was not immediately evident with the present passive avoidance task, we decided to retain the treatments and turn to other tasks. In retrospect, the present failure to observe inter- ference with passive avoidance is not surprising, since little "normal" forgetting of passive avoidance is ordinarily seen even over long retention periods. Our use of a passive avoidance task stemmed not from the expectation of "normal" forgetting, but from Calhoun et al.'s (1975) and Hinderliter et al.'s (1976) data suggesting that postECS sensory isolation following passive avoidance training decreases RA. It should be noted that this very lack of "normal" forgetting of passive avoidance poses problems for the application of Deutsch's hypothesis to the easily obtained RA for passive avoidance training that is induced by ECS.

\section{EXPERIMENT 3}

In Experiments 3 and 4, active avoidance was employed, rather than passive avoidance, because, presumably due to its response characteristics, active avoidance is not so readily acquired or retained. Thus, the potentially weaker memory for a small number of active avoidance training trials may be more susceptible to posttraining stimulation than was the memory for passive avoidance in the previous experiments. Using an active avoidance task, Experiment 3 sought ECS intensities that were just above and just below the threshold for producing RA. Then, in Experiment 4, interactions between this RA and post-ECS stimulation levels were sought.

\section{Method}

Subjects and Apparatus. Thirty-five rats, identical to those used in Experiments 1 and 2 except that they weighed 205-325 g, served as subjects. Training and testing occurred in a modified Jarvik-Kopp step-through apparatus constructed of Plexiglas in the shape of a 
vertical "V" with the bottom truncated. The apparatus was $63.5 \mathrm{~cm}$ in length, $28 \mathrm{~cm}$ in height, and $20 \mathrm{~cm}$ in width at the top, narrowing to $5.5 \mathrm{~cm}$ in width at the bottom. A sideways-moving opaque Plexiglas gate across the width divided the apparatus into two compartments. One compartment was $18 \mathrm{~cm}$ in length, painted white, and brightly illuminated by a $25-\mathrm{W}$ incandescent bulb mounted on the ceiling of the apparatus. The other compartment was $45.5 \mathrm{~cm}$ in length, dark in color, and unilluminated. The sloping side walls and floor of both compartments were composed of two bent metal plates. Centered between them at floor level was $1-\mathrm{cm}$ gap running the length of the compartment. Thus, the floor consisted of two $2.25-\mathrm{cm}$-wide plates separated by the $1-\mathrm{cm}$ gap. An animal was unable to stand on one plate without touching the other. The floor and walls of the small white compartment were used to deliver a $60-\mathrm{Hz}, 0.5-\mathrm{mA}$ constant-current footshock. When the gate was open, a hole, $8 \mathrm{~cm}$ in diameter and centered $5.5 \mathrm{~cm}$ above the floor, connected the two compartments. To add distinctiveness to the response of shuttling between compartments, a 4-cm hurdle blocked the bottom of the intercompartment hole. An adjacent ECS generator allowed administation of 300-msec ECS through earclips made from modified minigator clips. The time from opening of the gate to an animal's shuttling between compartments was automatically recorded.

Procedure. Each animal was transported in its home cage to the experimental room. After $30 \mathrm{sec}$ in the room, the animal was put in the white compartment. Three seconds later, the gate was opened. If the animal did not shuttle to the black compartment within $5 \mathrm{sec}$, footshock was administered until the subject escaped to the black compartment. Immediately after shuttling occurred, the gate was closed, and $3 \mathrm{sec}$ later the animal was returned to its home cage, which served as a holding cage. After $30 \mathrm{sec}$, another trial was given. This sequence was repeated for a total of five trials, on which the average animal avoided footshock 2.7 times. Any animal not avoiding on the fifth trial was eliminated from the study (five in all) and replaced with a naive subject. Upon completion of the fifth trial, the subjects immediately had earclips attached and were given ECS. (Time from shuttle response to ECS onset ranged from 6 to $10 \mathrm{sec}$.) Group assignments determined ECS intensity. Animals were assigned to groups $(n=7)$ based upon a counterbalancing of performance on Trials $1-4$ and body weight. the ECS intensities used were $0,25,35,45$, and $55 \mathrm{~mA}$. Approximately $72 \mathrm{~h}$ later, each animal in its home cage was returned to the experimental room and given one nonreinforced trial in which the gate was left open for $5 \mathrm{~min}$. Shuttle latencies from the opening of the gate and total time in the start compartment for each animal were recorded.

\section{Results and Discussion}

Shuttle latencies were transformed to log latencies to permit the use of parametric statistics. The normality of distributions of total times in the start compartment was not improved by either a log or a reciprocal transformation; therefore, statistical analysis was performed on total times in the start compartment and on log latencies for the initial shuttle response. Shuttle (log) latencies and total times in the start compartment exhibited the same basic relationships between groups, but within-group variances were somewhat less for total times in the start compartment. Hence, only these latter scores are reported here. Figure 3 depicts group means. Analysis of variance found significant differences between groups $[F(4,29)=$ 4.31, $\mathrm{p}<.01$ ]. [Pilot work had found that animals given pseudoconditioning (no footshock) spent a mean of about $160 \mathrm{sec}$ in the start compartment.] Although the $35-\mathrm{mA}$ ECS group spent a mean of only $128 \mathrm{sec}$ in the start compartment, comparison with the 45- and 55-mA groups in-

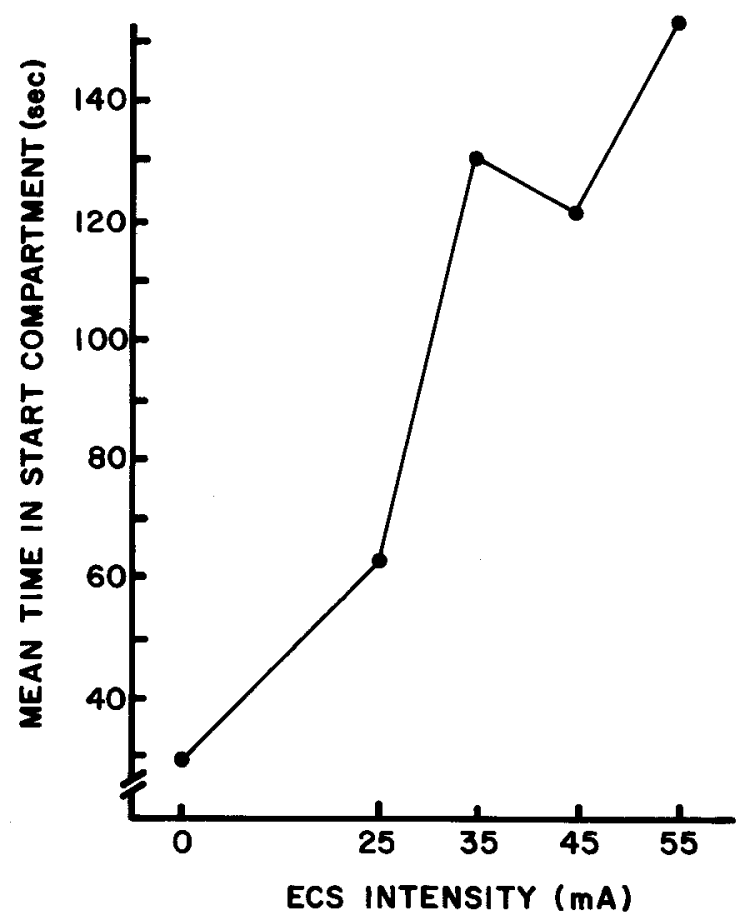

Figure 3. Mean time in start compartment on a 300-sec test as a function of ECS intensity following five active avoidance training trials.

dicated that increases in ECS added relatively little to test trial time in the start compartment. Hence, $35 \mathrm{~mA}$ was selected as the high-intensity ECS condition for Experiment 4 . For the low-intensity ECS condition, $25 \mathrm{~mA}$ was chosen despite its being only marginally different from $35 \mathrm{~mA}[\mathrm{t}(13)=1.93, \mathrm{p}<.1]$. Pilot studies had found that although lower values of ECS produced less RA, they also frequently failed to induce full seizures and also appreciably increased the likelihood of spinal damage.

\section{EXPERIMENT 4}

Like Experiment 2, Experiment 4 was designed to determine if post-ECS high stimulation would increase RA in animals that otherwise exhibited little or no RA due to low-intensity ECS, and if post-ECS low stimulation would decrease RA in animals that otherwise exhibited appreciable RA due to high-intensity ECS. As before, the high and low intensities of ECS were selected to avoid ceiling and floor effects that might mask the effects of post-ECS stimulation. Similarly, control groups were included to determine the impact of the high- and lowstimulation posttraining environments upon retention in animals not receiving ECS.

\section{Method}

Subjects and Apparatus. Forty-two animals of the same description as in the previous studies were used, except that body weights ranged from 175 to $300 \mathrm{~g}$. The training apparatus was the same as that used in Experiment 3, and the post-ECS environments were the same as those used in Experiment 2. 
Procedure. The procedure was identical to that of Experiment 3, except for ECS levels and the location in which the animals spent the hour after training. Moreover, all animals received 15 min of exposure to the low-stimulation enclosure for 3 days immediately preceding training. Counterbalancing for performance on Training Trials $1-4$ and body weight, the animals were assigned to one of seven groups $(n=6)$. Three groups received pseudo-ECS $(0 \mathrm{~mA})$ immediately after their fifth training trial, two groups received 25 $\mathrm{mA} E C S$, and two groups received $35-\mathrm{mA}$ ECS. One group receiving each ECS intensity was immediately returned to the home cage in the animal colony, one $0-\mathrm{mA}$ - and one $25-\mathrm{mA}$-ECS group were placed in the high-stimulation enclosure for $1 \mathrm{~h}$, and one $0-\mathrm{mA}$ and one 35-mA-ECS group were placed in the low-stimulation enclosure for $1 \mathrm{~h}$ before being returned to the home cage. Testing, identical to that in Experiment 3, occurred 3 days after training. The study was run in two balanced replications.

\section{Results and Discussion}

As in Experiment 3, within-group variances were lower for total test trial time in the start compartment than initial shuttle $(\log )$ latencies on the test trial, whereas the ordinal relationships between groups did not differ for the two behavioral measures. Hence, only total time in the start compartment is presented. Because the two replications yielded similar results, the data were pooled.

As can be seen in Figure 4, Groups 35-C, 25-C, and 0 -C yielded scores similar to those produced by equivalent groups in Experiment 3. Within ECS intensities, no differences between groups were observed (all ps $>.2$ ). Although far from significant, the ordering of the 0-mAECS groups was consistent, with posttraining low stimulation enhancing test trial performance and posttraining high stimulation impairing test trial performance, thus suggesting, in contrast to Experiment 2, some effect of posttraining stimulation. However, when the ECS groups are examined, any such effects appear to vanish rather than be magnified. Although the data are not supportive of the view that ECS increases susceptibility to ordinary sources of forgetting, as in Experiment 2 this could be attributed to a lack of appropriate stimulus interference. Adding subjects to the present study with the hope of obtaining significant differences at least between the 0-mA-ECS groups was considered, but a power analysis indicated that, assuming stable means and standard deviations, extremely large numbers of animals would be necessary. Consequently, the decision was made to turn to another task, presumably one that would be more readily influenced by the present posttraining stimulation conditions.

\section{EXPERIMENT 5}

One possible reason why the active avoidance task of Experiment 4 was relatively immune to disruption was that there were five training trials, with the critical ECS treatment being administered only after the last trial. Unfortunately, one-trial passive avoidance appears to be too well learned on a single trial to disrupt with modulation of posttraining environmental stimulation (Experiment 2), and the second training trial scores of Experiments 3 and 4 indicated that active avoidance is not learned sufficiently well on a single trial to provide sensitivity to ECS. However, one-trial appetitive learning has proven to be readily acquired and to be highly vulnerable to ECS (Tenen, 1965), and it might be expected to be more malleable by posttraining environmental stimulation than is onetrial passive avoidance, owing to presumably weaker motivation. Experiment 5 sought ECS values just above and just below the RA threshold for this task. Then Experi-

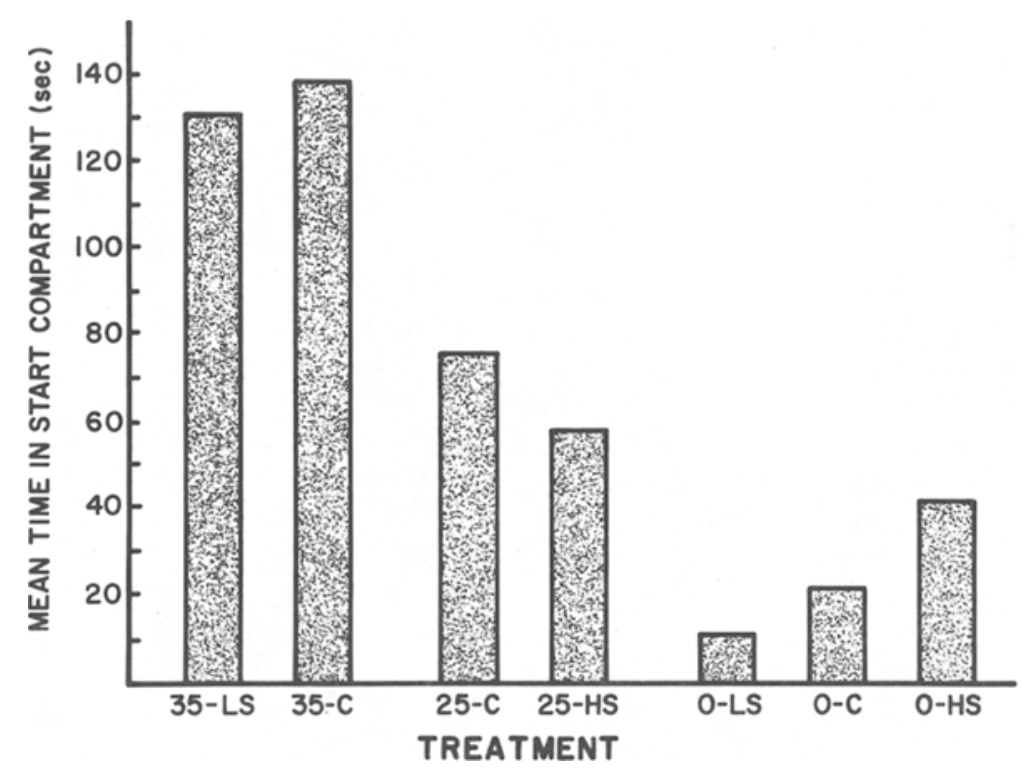

Figure 4. Mean time in start compartment after five active avoidance trials as a function of ECS intensity $(0,25$, or $35 \mathrm{~mA})$ and post-ECS stimulation (LS = low stimulation, $\mathrm{C}=$ home cage in animal colony, $\mathrm{HS}=$ high stimulation). 
ment 6 manipulated the post-ECS environment to determine if post-ECS stimulation interacted with ECS in determining the resultant RA.

\section{Method}

Subjects. The subjects were 41 rats identical to those used in the previous experiments, except that body weights ranged from 285 to $445 \mathrm{~g}$ and the subjects were water-deprived rather than fooddeprived. The water-deprivation schedule consisted of $10 \mathrm{~min}$ a day of access to water approximately $23 \mathrm{~h}$ prior to any experimental manipulation scheduled for the next day. Water deprivation was imposed gradually between 5 and 10 days before the experiment began. Water deprivation both kept the animals lean, thereby reducing the likelihood of ECS-induced spinal damage, and provided the motivation for the monitored behavior. Food was available ad lib in the home cage.

Apparatus. A $50.8 \times 50.8 \mathrm{~cm}$ open field enclosed by $57.2-\mathrm{cm}-$ high opaque walls was used for training and testing. The floor was composed of stainless steel grids. In one corner of the enclosure, a water-filled glass lick-tube protruded diagonally $1.25 \mathrm{~cm}$ into the enclosure. A lickometer circuit sensitive to resistance between the floor and the lick tube was used to monitor drinking. A pair of earclip electrodes, identical to those used in Experiments 1 and 2, were suspended from a counterweighted pulley system mounted directly over the center of the open field.

Procedure. Each animal had a pair of attached earclip electrodes attached in the home cage for $30 \mathrm{sec}$ per day on each of the 3 days preceding the experiment. This served to minimize the likelihood of the animals' resisting the earclips during training. On the training day, each animal had the suspended earclips affixed and was then placed in the center of the open field facing away from the lick tube. Actual search times took from 43 to $538 \mathrm{sec}$, except for one animal that was eliminated for not locating the lick tube within $600 \mathrm{sec}$. A second animal was eliminated for resisting the earclips. Upon locating the lick tube, the animals were allowed to 10 licks, a response that never took more than $3 \mathrm{sec}$, and then given ECS. One animal suffered spinal damage and consequently was removed from the study. This left 38 subjects in the study. Due to the unavailability of additional animals, the 3 subjects eliminated from the study were not replaced. ECS was $300 \mathrm{msec}$ of $0,25,35,45$, or $55 \mathrm{~mA}$. Group assignments were counterbalanced for time to locate the lick tube during training and for body weight. The final group sizes were $n=8(0,45$, and $55 \mathrm{~mA})$ and $n=7(25$ and $35 \mathrm{~mA}$ ). Three days after training, all subjects were returned to the training apparatus, just as on the training day except that earclips were not attached. Time to locate the lick tube and take 10 licks was recorded for each animal. A 600-sec ceiling was imposed, but no animal took more than $473 \mathrm{sec}$.

\section{Results and Discussion}

To improve the normality of the within-group distributions of test scores, all latencies were transformed to $\log$ latencies. Group mean log latencies are illustrated in Figure 5. With the exception of a slight inversion at $45 \mathrm{~mA}$, RA increased with ECS intensity $[\mathrm{F}(4,31)=$ $4.60, \mathrm{p}<.01]$.

Inspection of Figure 5 indicates that, with the present appetitive task, 25- and 35-mA ECS are just below and above the threshold for RA, respectively. The difference between Groups 0 and $35 \mathrm{~mA}$ was significant $[\mathrm{t}(13)=$ $2.37, \mathrm{p}<.05$ ], whereas the difference between Groups 0 and $25 \mathrm{~mA}$ was not $[\mathrm{t}(13)=1.08, \mathrm{p}<.2]$. Although the 25-mA group did not differ statistically from the 0 $\mathrm{mA}$ group, it clearly produced a tendency towards RA, such that a somewhat lower value of ECS would have been

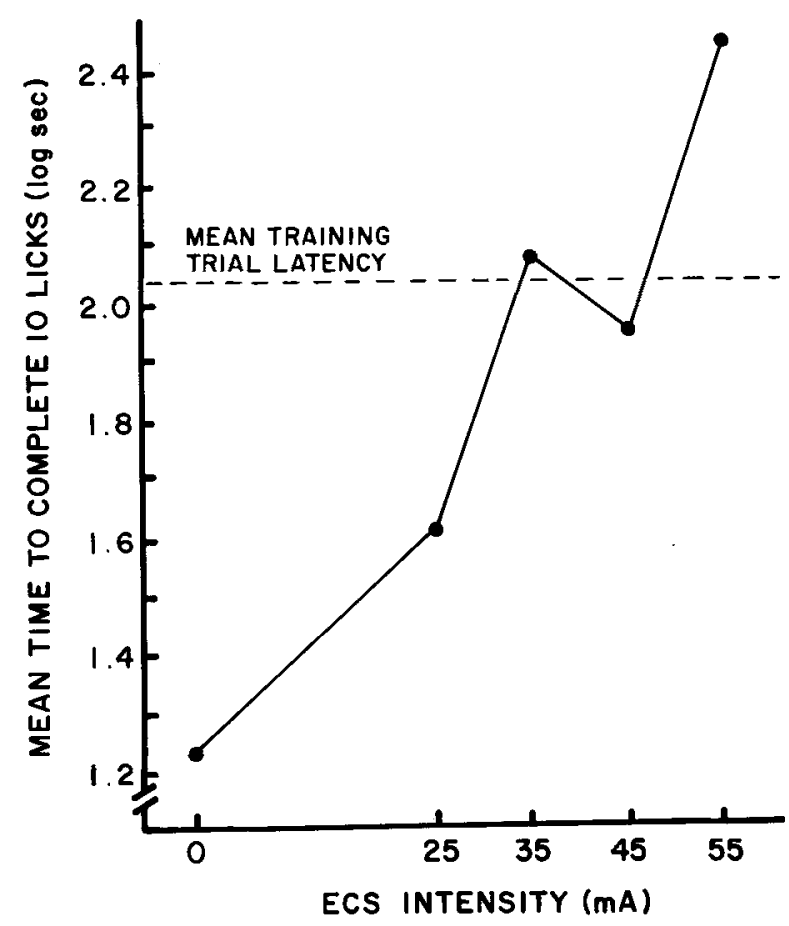

Figure 5. Mean latency to complete 10 licks as a function of ECS intensity following one appetitive training trial. The dotted line represents the mean training trial latency based on all animals.

preferred as the subthreshold intensity for RA. However, the same problems arose that were mentioned in Experiment 3. Weaker ECS both did not reliably produce full seizures and did increase the frequency of spinal damage. Similarly, 35-mA ECS yielded less RA than did $55 \mathrm{~mA}$, although the difference was not significant. Using $55 \mathrm{~mA}$ as the suprathreshold ECS value in Experiment 6 was rejected for fear of a ceiling effect, decreasing sensitivity to any RA-reducing effect of post-ECS sensory isolation. Notably, the ECS-induced active avoidance deficit observed in this experiment and Experiment 6 could, in principle, be attributed to the ECS's acting as a punisher rather than RA for active avoidance training (Coons \& Miller, 1960). However, numerous studies have demonstrated that a single ECS has no appreciable aversive value (e.g., McGaugh \& Madsen, 1964).

\section{EXPERIMENT 6}

As in Experiments 2 and 4, Experiment 6 investigated the effects of post-ECS low sensory stimulation upon RA induced by ECS just above the threshold for RA, and the effects of post-ECS high sensory stimulation upon RA induced by ECS just below the threshold for RA. The major difference between this and the earlier experiments was that this time a one-trial appetitive task was employed.

\section{Method}

Subjects and Apparatus. The subjects were 56 rats of the same description as that in the previous experiment, except that body weights ranged from 180 to $335 \mathrm{~g}$. The apparatus used for training 
and testing was the same as that used in Experiment 5. The highand low-stimulation enclosures were identical to those used in Experiments 2 and 4 , except that, due to an experimenter error, the room lights for the high-stimulation condition now flashed $0.5 \mathrm{sec}$ on $/ 0.5 \mathrm{sec}$ off.

Procedure. Except for ECS levels and the location in which animals recovered from ECS, the procedure was the same as that used in Experiment 5. Moreover, on each of the 3 days preceding training, all animals received $15 \mathrm{~min}$ of exposue to the lowstimulation enclosure. Counterbalancing for body weight and training trial latencies to complete 10 licks, subjects were assigned to one of seven groups. Upon completion of the 10 training licks, three groups of animals received 0-mA ECS (pseudo-ECS), two groups received 25-mA ECS, and two groups received 35-mA ECS. One group from each ECS intensity was then returned to the home cage in the colony room. One group from the 0 - and $35-\mathrm{mA}$ conditions spent $1 \mathrm{~h}$ in the low-stimulation enclosure immediately after ECS, and one group from the 0 - and 25-mA conditions spent $1 \mathrm{~h}$ immediately after ECS in the high-stimulation enclosure. All animals were tested 3 days after training, as in Experiment 5. The study was run in two replications.

\section{Results and Discussion}

Three animals were eliminated for removing their earclips during training, and two animals were eliminated during training for failing to complete 10 licks in less than $600 \mathrm{msec}$. These animals were not replaced, but the counterbalancing procedure allowed the losses to be distributed over groups. Thus, the effective group sizes were $\mathrm{n}=$ 8 for the two 25-mA groups and $n=7$ for the two 35$\mathrm{mA}$ groups and three 0 -mA groups. An analysis of variance, including a replication factor, found a replication effect $[F(1,37)=4.12, p<.05]$ but no interaction of replications with treatments $(\mathrm{p}>.5)$. The cause of the replication effect was not obvious, but to minimize withingroup variance in subsequent analyses, the replication factor was retained and planned comparisons between groups used the error term from the replication $x$ treatments analysis of variance.
Group mean log latencies to complete 10 licks are depicted in Figure 6. Consistent with Experiment 5, Groups $0-\mathrm{C}, 25-\mathrm{C}$, and 35-C yielded mean log latencies that increased as ECS levels increased, indicative of more intense ECS's causing greater RA. Both Group 35-C and Group 25-C differed from Group 0-C $[\mathrm{t}(12)=4.62$, $\mathrm{p}<.001$, and $\mathrm{t}(13)=2.47, \mathrm{p}<.05$, respectively]; however, Group 35-C did not yield significantly longer latencies than Group 25-C [t(13) $=1.53, \mathrm{p}>$.1]. No differences as a function of post-ECS stimulation were found between the 25-mA-ECS groups or between the 35$m A-E C S$ groups. To determine if this lack of effect was due to ECS's not interacting with post-ECS stimulation level, or to an inadequate variation of posttraining stimulation, the three 0-mA-ECS groups were examined. Although not significant, increasing stimulation (going from LS to $\mathrm{C}$ to $\mathrm{HS}$ ) yielded decreasing manifest retention of training; however, Group 0-LS did approach significance with respect to Group 0-HS $[\mathrm{t}(12)=1.82$, $.05<\mathrm{p}<.11$. The near significance of the effect of posttraining stimulation on the 0 -mA-ECS groups suggests that an interaction of ECS and post-ECS stimulation such as proposed by Deutsch (1973) and Hinderliter et al. (1976) should have been evident. However, the conclusion that there is no such interaction must be qualified by the lack of significance between the 0-mA-ECS groups. Adding subjects to the present study was considered, but a power analysis suggested that many additional subjects would be required to produce appreciable differences between the 0 -mA-ECS groups.

\section{EXPERIMENT 7}

Rather than adding animals to the one-trial appetitive task of Experiments 5 and 6, it was decided to switch to

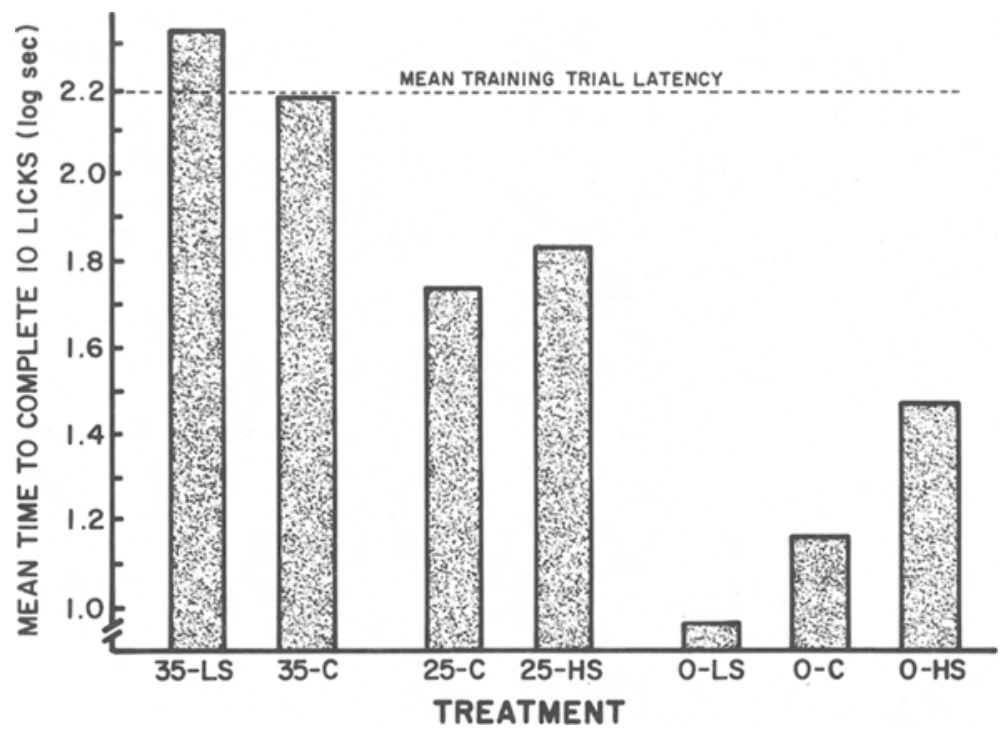

Figure 6. Mean latency to complete 10 licks after one-trial appetitive training as a function of ECS intensity $(0,25$, or $35 \mathrm{~mA})$ and post-ECS stimulation (LS = low stimulation, $\mathrm{C}=$ home cage in animal colony, $\mathrm{HS}=$ high stimulation). 
an appetitively motivated delayed alternation such as had been used by Gordon and Feldman (1976). Recent evidence obtained by Miller et al. (in press) suggests that test performance on this task is highly sensitive to the level of environmental stimulation during the retention interval. This task clearly incorporates one-trial learning while retaining the acquisition afforded by appetitively motivated tasks which is seemingly weaker, and hence potentially more disruptable than that afforded by aversively motivated tasks. Moreover, the target memory is presumably rendered more vulnerable than that of Experiments 5 and 6 , due to proactive interference between the current pair of trials (specific training and delay alternation test) and prior pairs of trials that occurred during general training on the task. Despite the laboriousness of teaching the general delayed alternation task to each subject, the possibility of giving an animal more than one critical pair of delayed alternation trials, that is, ECS and/or modified posttraining stimulation, was rejected because of the known acquired aversiveness of multiple ECSs (Coons \& Miller, 1960) and possible changes in the effectiveness of stimulation provided by the LS and HS environments as a function of repeated exposures to these enclosures. Because of the laborious training procedure, an estimation as to appropriate high and low ECS levels was made, and we immediately proceeded to look at the interaction of ECS intensity with post-ECS environmental stimulation. Control groups permitted a determination as to the appropriateness of the selected intensities of ECS.

\section{Method}

Subjects. One hundred and sixteen water-deprived rats of the same description as those used in Experiments 5 and 6 were used, except that body weights ranged between 185 and $385 \mathrm{~g}$ on Day 1 of the study.

Apparatus. The training and testing apparatus were two identical single-choice-point mazes. The mazes were initially E-mazes, but each was modified such that the start compartment was on the opposite side of the connecting transverse alley from the two goal compartments. One maze was used exclusively for the acquisition trials and the other maze was used for the test trials, thereby eliminating the possibility that on test trials animals would respond on the basis of odor trails formed during the preceding acquisition trial. In both mazes, the start compartment was $27.5 \mathrm{~cm}$ long, the transverse was $62 \mathrm{~cm}$ long, and the arms constituting the goalboxes were $19 \mathrm{~cm}$ long. All maze sections were $7 \mathrm{~cm}$ wide and $12.5 \mathrm{~cm}$ high, with stainless steel rods forming a grid floor. The walls, ceilings, and doors between compartments were made of clear Plexiglas. In each maze, at the far end of both goal compartments, was a glass dish $(4.5 \mathrm{~cm}$ in diameter and $1.5 \mathrm{~cm}$ deep) filled with water; however, access to the water in one of the two goal compartments in each maze was blocked on test trials by a clear Plexiglas barrier containing a number of .25 -cm-diam holes that allowed the passage of any odor that the water may have emitted. The walls of one goal compartment and the half of the transverse leading to it were externally covered with white paper, the walls of the other goal compartment and the other half of the transverse maze section were externally covered with black paper, and the start compartment walls were transparent. The experimental room was dimly illuminated, and a 70-dBC white noise served as an auditory background. Six identical holding cages, similar in construction to the subjects' home cages, were placed in the hallway just outside of the experimental room in order to restrain the animals during the delay intervals. The holding cages contained neither food nor water. In an adjacent room were the same high- and low-stimulation enclosures that were used in Experiment 2, 4, and 6.

Procedure. On Day 1, each water-deprived subject received 7.5 min of adaptation to each of the two mazes (all doors open), including unrestricted access to both of the water dishes in each maze. During this time, each animal visited all parts of the two mazes and drank from each water dish. Training began on Day 2 and consisted of a forced trial (target event) followed immediately by a free-choice trial. Forced trials consisted of placing an animal in the start compartment of the acquisition maze facing away from the choice point. At the choice point, a clear plastic door inserted perpendicular to the transverse maze section forced the animal to a goalbox that was chosen randomly for each animal on each forced trial. Upon reaching the accessible water dish, the animal was allowed to drink for $10 \mathrm{sec}$. Then, for the free-choice trial, it was placed in the start compartment of the test maze with access to both goal compartments, but with the perforated door blocking access to the water dish in the arm of the maze corresponding to the arm to which the animal had just been forced in the training maze; that is, alternation was rewarded. A choice was recorded when an animal's head reached the entrance to a goalbox; incomplete trips in the transverse prior to a choice were ignored. If the animal chose the reinforced arm, it was allowed to drink for $10 \mathrm{sec}$ and then returned to its home cage. However, if the animal failed to alternate with respect to the forced trial, it was given repeated correction trials without delay until it reached the accessible water dish and drank for $10 \mathrm{sec}$. A correction trial was procedurally identical to the immediately preceding test trial. After a delay of 15 to $30 \mathrm{~min}$ in the home cage had passed following a correct choice, another pair of forced and free trials was given. This continued until each animal had received a total of 10 pairs of trials.

Day 3 was identical to Day 2, except that each subject was placed in the holding cage for $30 \mathrm{sec}$ between forced and free trials. This continued over successive days until a subject responded correctly on 8 out of 10 consecutive free trials within a single day. Upon meeting this criterion, the delay interval was increased to $1 \mathrm{~min}$ and the number of trials was reduced to one pair per day. The 8out-of-10-correct criterion was simultaneously replaced by a 1 -outof-1-correct criterion, and the delay interval was increased on the next day. With each subsequent correct choice on the first free choice of the day, the delay interval was increased successively to $1.5 \mathrm{~min}$, then to $2,3,4,5,7.5,10,15,20,25$, and finally $30 \mathrm{~min}$. No correction trials were given after an animal responded correctly with the 2-min delay interval. Six subjects that were unusually slow in coming to exhibit consistent delayed alternation behavior were eliminated from the study during shaping. These animals had not reached the 5-min interval in 30 days, although the remaining 110 animals had reached this retention interval in an average of 19 days.

Upon reaching the 30-min retention interval, each subject was placed daily in a low-stimulation enclosure for 15 min beginning $30 \mathrm{~min}$ after its daily free-choice trial ( $30 \mathrm{~min}$ before receiving its daily ration of water). This manipulation was intended to habituate each animal to the low-stimulation enclosures. Daily pairs of forced and free trials continued with a 30-min delay interval until an animal made a correct choice on 5 consecutive days. The next day a probe trial pair occurred. The average animal reached its probe-trial pair after 28 days of running, including shaping.

The probe day differed from a nonprobe day in that the animal had earclips affixed and received 0-, 20-, or 35-mA ECS immediately after $10 \mathrm{sec}$ of drinking on the forced trial; then subjects were placed in either the high- or low-stimulation enclosures or the regular holding cage for $30 \mathrm{~min}$ before the free-choice trial. One animal was eliminated from the study due to spinal damage incurred at the time of ECS, and a second animal was eliminated because on the critical test trial it failed to leave the startbox within $10 \mathrm{~min}$ of placement there. Thus, 108 animals completed the experiment. 
These animals were assigned to one of six groups $(n=18)$, counterbalanced for body weight and number of days with a 30-min retention interval, that were required to reach the criterion of five consecutive correct choices. The six groups were 35-LS, 35-C, 20HS, 20-C, 0-LS, and 0-HS, where the numbers represent ECS intensity in milliamperes and the letters denote the post-ECS retentioninterval environment on the probe day, $\mathrm{C}$ denoting the regular holding cages. To maximize group size, no $0-\mathrm{C}$ group was included. Instead, the correctness of all 108 animals' choices on the 5th day that each subject was run with a 30 -min retention interval served as a measure of baseline performance. The experiment was performed in six nearly-balanced replications. Because of the small numbers of subjects in each treatment condition within each replication, no comparison across replications was attempted; similarly treated groups were simply pooled across replications.

\section{Results and Discussion}

The baseline $0-\mathrm{C}$ condition with 108 scores yielded 95 correct choices. With one choice made by each of 18 subjects, the number of correct choices were 17 for Group 0LS, 11 for Group 0-HS, 9 for Group 35-LS, 8 for Group 35-C, 12 for Group 20-C, and 13 for Group 20HS. Ratios of correct choices to total number of choices are illustrated in Figure 7.

To determine if there was an appreciable correlation within subjects between each animal's performance on the $0-\mathrm{C}$ baseline free-choice trial and its performance on the free-choice trial with modified stimulation during the retention interval, a contingency coefficient was calculated (Siegel, 1956). To reduce the number of cells with expected frequency values that were below five, Groups 0 -LS and 35-LS were pooled, Groups $20-\mathrm{C}$ and $35-\mathrm{C}$ were pooled, and Groups $0-\mathrm{HS}$ and $20-\mathrm{HS}$ were pooled. Moreover, within these three pooled groups, the few subjects that responded incorrectly on the $0-\mathrm{C}$ baseline trial were pooled independently of their responses on the ultimate test trial. The resultant 3 (LS, C, HS) $\times 3$ (ij = $11,10,01 / 00$, where $\mathrm{i}=1$ for correct or 0 for incorrect on the $0-\mathrm{C}$ baseline trial and $\mathrm{j}=1$ for correct or 0 for incorrect on the test trial) matrix yielded a correlation of $\mathrm{C}=0.16$. For comparison purposes, a correlation was calculated between each animal's performance on the 0 $\mathrm{C}$ baseline trial and the performance on the trial with modified stimulation during the retention interval of every other animal in the same group. To maintain comparability with the within-subject correlation, similar pooling of data was done. This between-subjects measure of correlation yielded a value of $C=0.14$. The high similarity of this between-subject correlation with the earlier withinsubject correlation prompted the use of statistics appropriate for independent groups in all cases, including comparisons of various groups with the $0-\mathrm{C}$ condition, in which all animals from each group were represented. (Miller et al., in press, also found a lack of within-subject correlation across trials on this task.) Because the study was initiated with the clear expectation (Deutsch, 1973; Spear, 1978) that ECS and HS treatments would impair performance if it had any effect at all, one-tailed statisti-

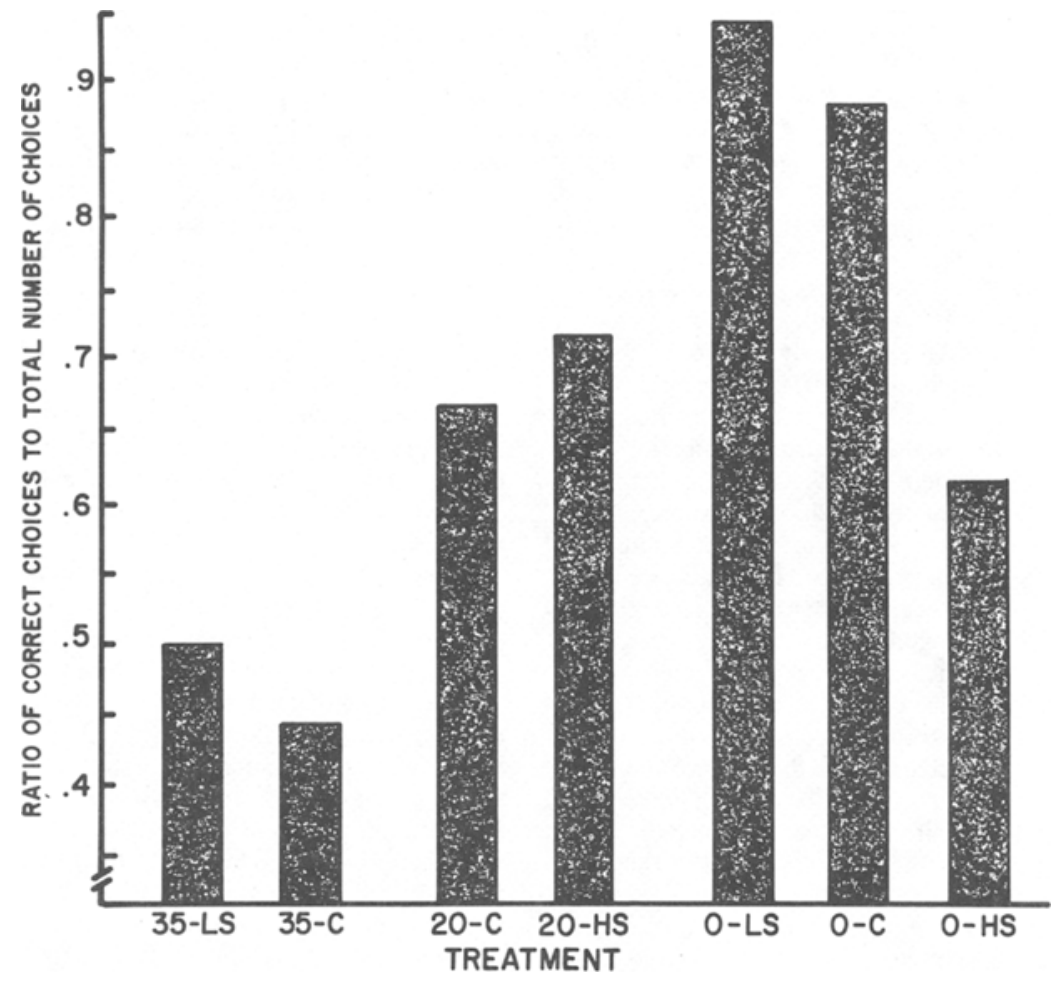

Figure 7. Ratio of correct choices to total number of choices as a function of ECS intensity (0, 25, or $35 \mathrm{~mA}$ ) and post-ECS stimulation ( $L S=$ low stimulation, $C=$ home cage in animal colony, $\mathrm{HS}=$ high stimulation). 
cal tests were used on all two-group comparisons.

Turning first to the effects of ECS alone, both Groups 20-C and 35-C performed significantly worse than animals in the $0-C$ condition $\left[\chi^{2}(1)=3.93, p<.025\right.$, and $\chi^{2}(1)$ $=16.77, \mathrm{p}<.0005$, respectively]. Group $20-\mathrm{C}$ did not differ from Group 35-C, but the nonsignificant tendency was towards more RA in Group 35-C, consisent with RA's being positively correlated with ECS intensity.

Examining the effects of HS and LS alone, Group 0LS showed a very weak (highly nonsignificant) tendency towards better retention than the animals in the $0-\mathrm{C}$ condition $(.5>p>.3)$, whereas Group 0-HS exhibited worse retention than the animals in the $0-\mathrm{C}$ condition $\left[\chi^{2}(1)=6.44, p<.01\right]$. Thus, retention on this delayed alternation task appears sensitive to high irrelevant environmental stimulation during the retention interval, HS being more disruptive than the normal retention interval environment.

Having determined that, with the present parameters, posttraining environmental stimulation was able to influence test performance, the critical question was whether, as Deutsch (1973) had suggested, ECS would amplify the performance-modifying influence of posttraining stimulation. Deutsch would predict that the effects of low-intensity ECS would be enhanced if high stimulation followed the ECS. However, comparing the performance of Group 20-C with that of Group 20-HS, it can be seen that the frequency of errors nonsignificantly decreased from 6 to 5 with exposure to high stimulation following ECS. Similarly, Deutsch would predict that the effects of high-intensity ECS would be attenuated if low stimulation followed the ECS. Although a comparison of Group 35-C with Group 35-LS does indicate that the frequency of errors nonsignificantly decreased from 10 to 9 with exposure to low stimulation following ECS, both groups were clearly performing at chance. This latter outcome may have reflected a ceiling effect for RA produced by too intense an ECS. But the lack of a significant difference between the 20-mA groups is clearly free of any possible ceiling or floor effects.

\section{GENERAL DISCUSSION}

Using four distinctly different learning tasks, one-trial passive avoidance (Experiment 2), multitrial active avoidance (Experiment 4), one-trial active approach (Experiment 6), and "one-trial" appetitive delayed alternation (Experiment 7), efforts were made to enhance, with ECS, the impact upon test performance of posttraining environmental stimulation with little in common with the target task. Although some of the studies employed numbers of subjects too small to achieve significance between ECS and non-ECS groups, none of the four studies provided so much as a nonsignificant tendency in the direction of Deutsch's (1973) hypothesis. Instead, the experiments either yielded no consistent tendencies or (nonsignificant) tendencies in the opposite direction; that is, tendencies towards ECS and subsequent high environmen- tal stimulation yielding less disruption of test performance than would be expected from the algebraic summation of environmental stimulation alone and ECS alone.

Collectively, these studies support an inductive argument against the hypothesis that ECS potentiates environmental sources of forgetting. However, any such conclusion has to be tempered due to the limited impact of the present high- and low-stimulation conditions in the absence of ECS. If ECS did enhance disruption of performance resulting from posttraining stimulation, significant effects of posttraining stimulation without ECS would be necessary to ensure sensitivity to the hypothesized effect. In the present four tasks (Experiments 2, 4, 6, and 7), greater interference with performance as a result of increased posttraining stimulation was not evident with the passive avoidance task (despite this being the task used by Hinderliter et al., 1976), was suggested but nonsignificant with the active avoidance and appetitive approach tasks, and achieved statistical significance only in the case of the appetitively motivated delayed alternation task. One factor that may have rendered delayed alternation particularly susceptible to high environmental stimulation during the retention interval is that the many pairs of forced and free-choice trials given each animal in preparation for the critical pair of trials possibly left long-term memory highly subject to intertrial proactive interference, thereby forcing upon the animal a greater dependency on short-term memory than would be the case with the three earlier tasks. If short-term memory were of special import to the delayed alternation task relative to the prior tasks and the effect of posttraining stimulation alone depended upon this factor, then generalizing the present rather decisive refutation of Deutsch's (1973) hypothesis from delayed alternation to other tasks would be suspect. However, to the extent that the null results of Experiments 2,4 , and 6 speak to the issue, the present data do argue against ECS's merely enhancing the impact of conventional sources of forgetting. Rather, the current results are far more compatible with the view that ECS is a primary source of disruption in its own right. The nature of the mechanism by which it produces this disruption is still to be determined.

\section{REFERENCES}

Adams, H. E., Calhoun, K. S., Davis, J. W., \& Peters, R. D. (1974). Effects of isolation on retrograde amnesia produced by ECS in multiple trial learning. Physiology \& Behavior, 12, 499-501.

Calhoun, K. S., Prewett, R. D., Peters, R. D., \& Adams, H. E. (1975). Factors in the modification by isolation of electroconvulsive shock-produced retrograde amnesia in the rat. Journal of Comparative and Physiological Psychology, 88, 373-377.

Coons, E. E., \& Miller, N. E. (1960). Conflict versus consolidation of memory traces to explain "retrograde amnesia" produced by ECS. Journal of Comparative and Physiological Psychology, 53, 524-531.

DEUTSCH, J. A. (1973). Electroconvulsive shock and memory. In J. A Deutsch (Ed.), The physiological basis of memory (pp. 113-124). New York: Academic Press.

GolD, P. E., \& STERNBERG, D. B. (1978). Retrograde amnesia produced by several treatments: Evidence for a common neurobiological mechanism. Science, 201, 367-369.

Gordon, W. C., \& FeldMAN, D. T. (1976). Reactivation-induced in- 
terference in a short-term retention paradigm. Learning and Motivation, 9, 164-178.

Hinderliter, C. F., Smith, S. L., \& Misanin, J. R. (1976). A reduction of ECS-produced amnesia through post-ECS sensory isolation. Bulletin of the Psychonomic Society, 7, 542-544.

MARLin, N. A., Greco, C., \& Miller, R. R. (1978). Effects of posttraining reinforcement upon retention of a passive avoidance task. Bulletin of the Psychonomic Society, 11, 295-297.

MCGAUGH, J. L. (1966). Time-dependent processes in memory storage. Science, 153, 1351-1358.

McGaugh, J. L., \& Madsen, M. C. (1964). Amnesia and punishing effects of electroconvulsive shock. Science, 144, 182-183.

Mrller, R. R., Greco, C., Marlin, N. A., \& Balaz, M. A. (in press). Retroactive interferences in rats: Independent effects of time and similarity of the intervening event with respect to acquisition. Quarterly Journal of Experimental Psychology.

Miller, R. R., \& MARLIN, N. A. (1979). Amnesia following electroconvulsive shock. In J. F. Kihlstrom \& F. J. Evans (Eds.), Disorders of memory function (pp. 143-178). Hillsdale, NJ: Erlbaum.

MilleR, R. R., \& SPRINGER, A. D. (1972). Induced recovery of memory in rats following electroconvulsive shock. Physiology \& Behavior, 8, 645-651.

Pagano, R. R., Bush, D. F., Martin, G., \& Hart, E. B. (1969). Duration of retrograde amnesia as a function of electroconvulsive shock intensity. Physiology \& Behavior, 4, 19-21.

Peters, R. D., Calhoun, K. S., \& Adams, H. E. (1973). Modification by environmental conditions of retrograde amnesia produced by ECS. Physiology \& Behavior, 11, 889-892.

SIEGEL, S. (1956). Nonparametric statistics for the behavioral sciences. New York: McGraw-Hill.

SMIth, G. J., \& SPEAR, N. E. (1979). Reactivation of an appetitive discrimination memory following retroactive interference. Animal Learning \& Behavior, 7, 289-293.

SPEAR, N. E. (1978). The processing of memories: Forgetting and retention. Hillsdale, NJ: Erlbaum.

TENEN, S. S. (1965). Retrograde amnesia from electoconvulsive shock in a one-trial appetitive learning task. Science, 148, 1248-1250.

(Manuscript received July 2, 1984;

revision accepted for publication January 20, 1985.) 